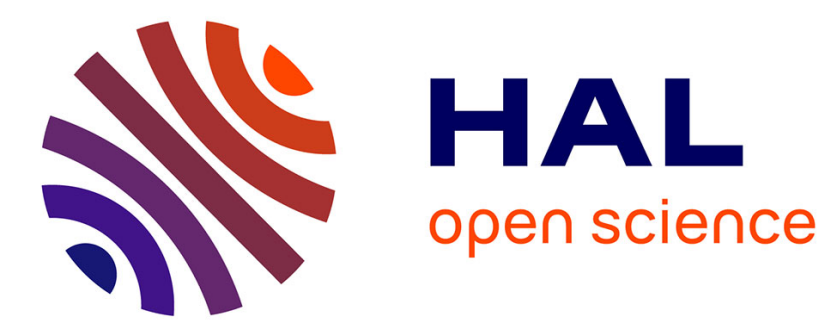

\title{
Integrator forwarding via dynamic scaling
}

\author{
Laurent Praly, D. Carnevale, Alessandro Astolfi
}

\section{To cite this version:}

Laurent Praly, D. Carnevale, Alessandro Astolfi. Integrator forwarding via dynamic scaling. 49th IEEE Conference on Decision and Control, Dec 2010, Atlanta, United States. pp.5955-5960, 10.1109/CDC.2010.5717175 . hal-00554865

\section{HAL Id: hal-00554865 \\ https: / hal-mines-paristech.archives-ouvertes.fr/hal-00554865}

Submitted on 11 Jan 2011

HAL is a multi-disciplinary open access archive for the deposit and dissemination of scientific research documents, whether they are published or not. The documents may come from teaching and research institutions in France or abroad, or from public or private research centers.
L'archive ouverte pluridisciplinaire HAL, est destinée au dépôt et à la diffusion de documents scientifiques de niveau recherche, publiés ou non, émanant des établissements d'enseignement et de recherche français ou étrangers, des laboratoires publics ou privés. 


\title{
Integrator forwarding via dynamic scaling
}

\author{
L. Praly, D. Carnevale, A. Astolfi
}

\begin{abstract}
The problem of integrator forwarding is revisited using the notion of dynamically scaled (control) Lyapunov function. A new class of dynamic stabilizing control laws is presented, and connections with classical forwarding are established.
\end{abstract}

\section{INTRODUCTION}

The problem of stabilization for general nonlinear systems using control Lyapunov functions has been recently revisited in [7], on the basis of the ideas introduced in [4]. In particular, the main result of [7] states that, under suitable technical assumptions, the existence of a statically scaled control Lyapunov function, i.e. of a control Lyapunov function of the form

$$
l_{1}\left(V_{1}\right)+l_{2}\left(V_{2}\right)
$$

with $l_{1}$ and $l_{2}$ scaling functions, is equivalent to the existence of a dynamically scaled control Lyapunov function, i.e. of a function of the form

$$
V_{1}+\frac{V_{2}}{r}
$$

where $r$ is a dynamic parameter which is updated on-line.

A direct consequence of this equivalence result is that control design, exploiting for example universal formulae [8], [2], can be performed using either the statically scaled function or the dynamically scaled one. This observation leads naturally to the study of the advantages and disadvantages of these two designs. A few comments in this direction have been already given in [7], where it is noted that the design based on the dynamically scaled Lyapunov function is simpler in that it does not require the explicit construction of the scaling functions $l_{1}$ and $l_{2}$, although it requires the design of the differential equation of the scaling parameter $r$, which may be non-trivial. In addition, in [4] it has been shown that the use of dynamically scaled Lyapunov functions is helpful in some adaptive control and observer design problems, since the dynamics of $r$ can be specified without the knowledge of unknown parameters or unmeasured states.

Goal of this paper is to exploit dynamically scaled control Lyapunov functions in the design of stabilizing control

L. Praly is with MINES ParisTech, CAS, Mathématiques et Systèmes, Fontainebleau, France, E-mail: Laurent.Praly@mines-paristech.fr

D. Carnevale is with DISP, University of Roma "Tor Vergata", Via del Politecnico 1, 00133 Rome, Italy, E-mail: carnevale@disp.uniroma2.it

A. Astolfi is with the Dept. of Electrical and Electronic Engineering, Imperial College London, London, SW7 2AZ, UK and DISP, University of Roma "Tor Vergata", Via del Politecnico 1, 00133 Rome, Italy, E-mail: a.astolfi@ic.ac.uk

This work is partly supported by the EPSRC Programme Grant "Control For Energy and Sustainability" EP/G066477 and by the MIUR under PRIN Project "Advanced Methods for Feedback Control of Uncertain Nonlinear Systems". laws for a class of feedforward systems (in line with the preliminary results in [1]) and to highlight the amount of prior knowledge required in the design of the dynamics of the scaling parameter. In addition, it is shown that the design based on dynamic scaling allows to partly match the gain of any linear feedback, designed for the linear approximation of the system around the origin.

We complete the introduction noting that dynamic scaling has been used in the design of control laws for feedforward systems in [11], [5]. In [11] stabilizing control laws based on Teel's nested saturation scheme [9], [10] and on the introduction of a state scaling parameter have been designed. The control design relies upon the definition of scaled dynamics and the dynamic scaling parameter is monotonically updated using a logic-based scheme. Similarly, in [5] scaled dynamics are defined, whereas the dynamics of the scaling parameter obey a differential equation. There are some significant differences with the approach put forward in this paper. First, in the present paper it is the Lyapunov function, and not the state, which is scaled. Secondly, and most importantly, in this paper the extended system, i.e. the system with state including the dynamic scaling parameter, has a globally, asymptotically, stable equilibrium, whereas in [11], [5] boundedness of the dynamic scaling parameter is assessed a-posteriori and relies upon convergence to zero of the state of the underlying system.

\section{Notes}

1) We use the verb know to mean that the corresponding object is available to the designer, whereas we use the verb exist to mean that the corresponding object exists but it cannot be used by the designer.

2) To help the reader we collect here some definitions which are used throughout the paper:

$$
\begin{aligned}
z & =y-M(x), \\
\delta(x) & =h(x)-L_{f} M(x), \\
U(x, y, r) & =V(x)+\frac{z^{2}}{r}, \\
N\left(x, \frac{z}{r}\right) & =L_{g} V(x)-\frac{2 z}{r} L_{g} M(x), \\
P\left(x, \frac{z}{r}\right) & = \\
L_{g} M(x) L & \left(x, \frac{z}{r}\right)\left(K(x)+L\left(x, \frac{z}{r}\right)\right)^{-1} K(x) L_{g} M(x)^{T}
\end{aligned}
$$




\section{Preliminaries AND PROBlem DEFINITION}

Consider systems described by equations of the form

$$
\left\{\begin{array}{l}
\dot{y}=h(x), \\
\dot{x}=f(x)+g(x) u,
\end{array}\right.
$$

where $x$ is in $\mathbb{R}^{n}, y$ is in $\mathbb{R}$ and $u$ is in $\mathbb{R}^{m}$. The functions $h$ and $f$ are $C^{1}$ and zero at the origin and the function $g$ is continuous. System (4) is one of the simplest feedforward forms. For such a system static, state feedback, stabilizing control laws can be designed using several approaches, see for example [10], [6], [3]. Unlike these papers, we aim at designing a dynamic, state feedback, control law of the form

$$
\dot{r}=\psi(x, y, r), \quad u=\phi(x, y, r),
$$

with $r$ in $^{1} \mathbb{R}_{+*}$, which renders the point $\left(0,0, r_{*}\right)$, for some $r_{*}>0$, asymptotically stable (see Section III). To achieve this goal we first revisit the classical forwarding construction of [6].

\section{A. Forwarding revisited}

In [6] it is shown that, static, globally stabilizing control laws for system (4) exist provided the designer knows a $C^{1}$ positive definite and radially unbounded function $V$ and a $C^{1}$ function $M$ such that ${ }^{2}$ :

- the functions ${ }^{3}$ :

$$
\begin{aligned}
& x \mapsto-L_{f} V(x), \\
& x \mapsto W(x):=-L_{f} V(x)-L_{g} V(x) K(x) L_{g} V(x)^{T}(6)
\end{aligned}
$$

are continuous and positive definite, where $K$ is a $C^{0}$ function, the values of which are non-negative symmetric matrices ${ }^{4}$;

$$
\begin{gathered}
\text { - } \quad \limsup _{|x| \rightarrow 0} \frac{\left|h(x)-L_{f} M(x)\right|}{W(x)}<+\infty, \\
\text { - } \quad L_{g} M(0) \neq 0 .
\end{gathered}
$$

Let $\ell$ and $m$ be two $C^{1}$ class $\mathcal{K}^{\infty}$ functions, define the function

$$
\mathfrak{V}(x, y)=\ell(V(x))+m\left((y-M(x))^{2}\right)
$$

and note that its derivative along solutions is

$$
\begin{aligned}
\overparen{\mathfrak{V}(x, y)}= & -\ell^{\prime}(V(x))\left|L_{f} V(x)\right|+2 m^{\prime}\left(z^{2}\right) z \delta(x) \\
& +\left[\ell^{\prime}(V(x)) L_{g} V(x)-2 m^{\prime}\left(z^{2}\right) z L_{g} M(x)\right] u,
\end{aligned}
$$

where, as defined in (3) we have used the notation

$$
z=y-M(x), \quad \delta(x)=h(x)-L_{f} M(x) .
$$

\footnotetext{
${ }^{1} \mathbb{R}_{+*}$ denotes the set of strictly positive real numbers.

${ }^{2}$ This assumption holds when the origin is a globally asymptotically and locally exponentially stable equilibrium and $\frac{\partial h}{\partial x}(0) \frac{\partial f}{\partial x}(0)^{-1} g(0)$ is not zero.

${ }^{3}$ This condition can be enforced with the preliminary feedback $u=$ $-K(x) L_{g} V(x)^{T}$, with $K$ designed so that this feedback satisfies in a strict way any given constraint on the control.

${ }^{4}$ It is sufficient that only its symmetric part be non-negative.
}

As a result, the function $\mathfrak{V}$ is a control Lyapunov function provided

$$
\begin{aligned}
2 m^{\prime}\left(z^{2}\right) z \delta(x) & <\ell^{\prime}(V(x))\left|L_{f} V(x)\right| \\
\forall(x, z) & \neq 0: 2 m^{\prime}\left(z^{2}\right) z L_{g} M(x)=\ell^{\prime}(V(x)) L_{g} V(x) .
\end{aligned}
$$

Conditions (9) hold for any functions $\ell$ and $m$ if $\delta$ is identically zero, which means that we know the function $M$ solution of :

$$
L_{f} M(x)=h(x) .
$$

When $\delta$ is not identically zero, conditions (9) hold if either of the following conditions holds.

1) For all non-zero $x$ satisfying :

$$
L_{g} M(x) \neq 0, \quad \frac{L_{g} V(x)}{L_{g} M(x)} \delta(x)>\left|L_{f} V(x)\right|,
$$

we have that $\frac{L_{g} V(x)}{L_{g} M(x)} \ell^{\prime}(V(x))$ is not in the image of the function $z \mapsto 2 z m^{\prime}\left(z^{2}\right) . \mathrm{s}$

2) For all non-zero $x$ satisfying

$$
L_{g} V(x)=L_{g} M(x)=0,
$$

any $\mu$ that is in the image of the function $z \mapsto$ $2 z m^{\prime}\left(z^{2}\right)$ satisfies

$$
\mu \delta(x)<\ell^{\prime}(V(x))\left|L_{f} V(x)\right| .
$$

More conservatively, conditions (9) are satisfied if we choose the functions $\ell$ and $m$ satisfying :

$$
\frac{2|z||\delta(x)|}{\left|L_{f} V(x)\right|}<\frac{\ell^{\prime}(V(x))}{m^{\prime}\left(z^{2}\right)} \quad \forall x \neq 0 .
$$

By condition (7) and since $V$ is radially unbounded, it is always possible to select $\ell$ and $m$ such that condition (11) holds. The selection relies upon the following statement established in [6] and the proof of which is given in the appendix for convenience.

Lemma 1: Under condition (7), there exists a $C^{1}$ function $\ell: \mathbb{R}_{+} \rightarrow \mathbb{R}_{+}$with non-decreasing and non-integrable derivative $\ell^{\prime}$ satisfying :

$$
|\delta(x)| \leq \ell^{\prime}(V(x))\left|L_{f} V(x)\right| \quad \forall x \in \mathbb{R}^{n} .
$$

Once $\ell$ has been selected as in Lemma 1, the selection

$$
m(s)=\sqrt{1+s}-1
$$

is such that condition (11) holds.

\section{B. Motivating dynamic scaling}

The left hand side of equation (11) is $x$ dependent, whereas the right hand side is $V(x)$ dependent. This means that, in general, there is no tight solution to the inequality (11), whereas we are interested in a tight solution. The main reason for the satisfaction of the tight inequality is as follows. The "nominal" feedback resulting from the use of the statically scaled control Lyapunov function is of the form :

$u=-2 L_{0}(x, z)\left[\ell^{\prime}(V(x)) L_{g} V(x)-2 z m^{\prime}\left(z^{2}\right) L_{g} M(x)\right]^{T}$, 
with $L_{0}$ a continuous function, the values of which are positive definite symmetric matrices ${ }^{5}$. In this feedback the contribution of $z$ is reduced by the fact that $z m^{\prime}\left(z^{2}\right)$ is bounded (see (11)) and $\ell^{\prime}(V(x))$ is larger.

Finally note that, around the origin, the nominal control is approximated by

$$
u \approx-2 L_{0}(0,0)\left[\ell^{\prime}(0) x^{T} \frac{\partial^{2} V}{\partial x^{2}}(0) g(0)-\frac{\partial M}{\partial x}(0) g(0) z\right]^{T}
$$

where according to $(12), \ell^{\prime}(0)$ is constrained by the inequality

$$
\ell^{\prime}(0) \geq \limsup _{x \rightarrow 0} \frac{|\delta(x)|}{\left|L_{f} V(x)\right|} .
$$

Since $\ell^{\prime}(0)$ dictates the relative importance of the $z$-term in the approximation of the control around the origin, it may be difficult to select $\ell$ to match the gain of $z$ of a (given) linear stabilizing feedback (designed on the linear approximation of the system around the origin).

To possibly increase the contribution of $z$ in the feedback, we exploit the idea in [1] and we take advantage of the dynamic extension $\dot{r}=\psi$, with $r$ taking values in $\mathbb{R}_{+*}$. As in [1], $r$ plays the role of the ratio $\frac{\ell^{\prime}(V(x))}{m^{\prime}(z)}$.

This leads to consider the partial control Lyapunov function (see [7]):

$$
U(x, y, r)=V(x)+\frac{z^{2}}{r} .
$$

We use the term partial since $U$ is positive definite and proper in $x$ and $\frac{z}{\sqrt{r}}$ only.

\section{MAIN RESULTS}

In this section we employ the partial control Lyapunov function (15) to design dynamic stabilizing control laws of the form (5). We present three alternative designs, each relying on a set of data on the system (4) and on the scaling function $\ell$.

\section{A. Design I - $\ell$ known, $K$ non-positive definite.}

The simplest design relies on the knowledge of the function $\ell$ satisfying the condition of Lemma 1. Precisely, suppose we know a $C^{1}$ function $\kappa: \mathbb{R}_{+} \rightarrow \mathbb{R}_{+}$, which is zero at zero, with non-decreasing and non-integrable derivative $\kappa^{\prime}$, satisfying :

$$
\frac{2|\delta(x)|}{\left|L_{f} V(x)\right|}<\sqrt{\kappa^{\prime}(V(x))} \quad \forall x \in \mathbb{R}^{n} \backslash\{0\} .
$$

Define the function

$$
\begin{aligned}
\mathcal{U}(x, y, r)=\left[r-r_{*} \log (r)-r_{*}+r_{*}\right. & \left.\log \left(r_{*}\right)\right] \\
& +\frac{\kappa(U(x, y, r))}{\alpha},
\end{aligned}
$$

with $\alpha \in(0,1)$ and $r_{*}>0$ design parameters. Note that $\mathcal{U}$ is positive definite in $\left(x, y, r-r_{*}\right)$ and proper on $\mathbb{R}^{n} \times \mathbb{R} \times \mathbb{R}_{+*}$. Its derivative along the solutions of system

\footnotetext{
${ }^{5}$ Again it is sufficient that only the symmetric parts be positive definite.
}

(4)-(5) is :

$\overparen{\mathcal{U}(x, y, r)}=\frac{r-r_{*}}{r} \psi$
$+\frac{\kappa^{\prime}(U(x, y, r))}{\alpha}\left[L_{f} V(x)+\frac{2 z \delta(x)}{r}+N\left(x, \frac{z}{r}\right) u-\frac{z^{2}}{r^{2}} \psi\right]$,

where

$$
N\left(x, \frac{z}{r}\right)=L_{g} V(x)-\frac{2 z}{r} L_{g} M(x) .
$$

Selecting the nominal control

$$
u=\phi\left(x, \frac{z}{r}\right)=-2 L\left(x, \frac{z}{r}\right) N\left(x, \frac{z}{r}\right)^{T},
$$

where $L$ is a continuous function, the values of which are positive definite symmetric matrices, yields

$$
\begin{aligned}
\overparen{\mathcal{U}(x, y, r)} & =\left(\frac{r-r_{*}}{r}-\frac{\kappa^{\prime}(U(x, y, r))}{\alpha} \frac{z^{2}}{r^{2}}\right) \psi \\
& -\frac{\kappa^{\prime}(U(x, y, r))}{\alpha}\left[\left|L_{f} V(x)\right|-\frac{2 z \delta(x)}{r}\right] \\
& -2 \frac{\kappa^{\prime}(U(x, y, r))}{\alpha} N\left(x, \frac{z}{r}\right) L\left(x, \frac{z}{r}\right) N\left(x, \frac{z}{r}\right)^{T},
\end{aligned}
$$

where using (16) and the property that $\kappa^{\prime}$ is non decreasing, we have :

$$
\begin{aligned}
\frac{2|z \delta(x)|}{r} & -\sqrt{\alpha}\left|L_{f} V(x)\right| \\
\leq & \sqrt{\alpha}\left|L_{f} V(x)\right|\left[\sqrt{\left.\frac{\kappa^{\prime}(V(x))}{\alpha} \frac{|z|}{r}-1\right],}\right. \\
\leq & \sqrt{\alpha}\left|L_{f} V(x)\right|\left[\sqrt{\frac{\kappa^{\prime}(U(x, y, r))}{\alpha}} \frac{|z|}{r}-1\right], \\
\leq & \alpha\left|L_{f} V(x)\right| \frac{\left[\frac{\kappa^{\prime}(U(x, y, r))}{\alpha} \frac{|z|^{2}}{r^{2}}-\frac{r-r_{*}}{r}\right]-\frac{r_{*}}{r}}{\sqrt{\kappa^{\prime}(V(x))}} \frac{|z|}{r}+\sqrt{\alpha}
\end{aligned}
$$

Hence, with $\psi$ as control, the function $\mathcal{U}$ is a control Lyapunov function satisfying the small control property. It follows that $\psi$ can be selected using one of the universal formulae [8], [2]. For instance, selecting

$$
\psi= \begin{cases}-\frac{A+\sqrt{A^{2}+B^{4}}}{B} & \text { if } B \neq 0 \\ 0 & \text { if } B=0\end{cases}
$$

where :

$$
\begin{aligned}
A & =-\frac{\kappa^{\prime}(U(x, y, r))}{\alpha}\left[\sqrt{\alpha}\left|L_{f} V(x)\right|-\frac{2 z \delta(x)}{r}\right], \\
B & =\frac{r-r_{*}}{r}-\frac{\kappa^{\prime}(U(x, y, r))}{\alpha} \frac{z^{2}}{r^{2}},
\end{aligned}
$$

yields

$$
\begin{aligned}
\overparen{\mathcal{U}(x, y, r)} & \leq-\left(\frac{r-r_{*}}{r}-\frac{\kappa^{\prime}(U(x, y, r))}{\alpha} \frac{z^{2}}{r^{2}}\right)^{2} \\
& -\frac{1-\sqrt{\alpha}}{\alpha} \kappa^{\prime}\left(U(x, y, r)\left|L_{f} V(x)\right|\right. \\
& -2 \frac{\kappa^{\prime}(U(x, y, r))}{\alpha} N\left(x, \frac{z}{r}\right) L\left(x, \frac{z}{r}\right) N\left(x, \frac{z}{r}\right)^{T} .
\end{aligned}
$$

Hence $\overparen{\mathcal{U}(x, y, r)}$ is negative definite in $\left(x, y, r-r_{*}\right)$ on $\mathbb{R}^{n} \times$ $\mathbb{R} \times \mathbb{R}_{+*}$. This establishes that the dynamic feedback given 
by (18), (19) is asymptotically stabilizing the point $\left(0,0, r_{*}\right)$ with $\mathbb{R}^{n} \times \mathbb{R} \times \mathbb{R}_{+*}$ as domain of attraction.

Finally note that, close to this equilibrium point, the control can be approximated by

$$
u \approx-2 L(0,0)\left[x^{T} \frac{\partial^{2} V}{\partial x^{2}}(0) g(0)-\frac{\partial M}{\partial x}(0) g(0) \frac{2 z}{r_{*}}\right]^{T}
$$

where as opposed to $\ell^{\prime}(0)$ in (14), $r_{*}$ is a free design parameter which may be selected to adjust the gain multiplying $z$, and hence to possibly recover a linear feedback that has been designed for the linearized system around the origin.

\section{B. Design II - - unknown, $K$ positive definite}

In this section we deal with the case in which the function $\ell$ is unknown (although it exists). Let $u$ be as in (18). Using (6), we have

$$
\begin{aligned}
\overparen{U(x, y, r)}= & -W(x)+\frac{2 z \delta(x)}{r}-\frac{z^{2}}{r^{2}} \psi \\
& -2 N\left(x, \frac{z}{r}\right) L\left(x, \frac{z}{r}\right) N\left(x, \frac{z}{r}\right)^{T} \\
& -L_{g} V(x) K(x) L_{g} V(x)^{T}
\end{aligned}
$$

where, by (6) and (12), we have, for all $\alpha \in(0,1)$,

$$
\begin{aligned}
-\alpha W(x)+\frac{2 z \delta(x)}{r} & \leq \frac{\delta(x)^{2}}{\alpha W(x)} \frac{z^{2}}{r^{2}} \\
& \leq \frac{\ell^{\prime}(V(x))^{2}}{\alpha} W(x) \frac{z^{2}}{r^{2}} .
\end{aligned}
$$

So, with $\psi$ as control, $U$ is a partial control Lyapunov function which satisfies the small control property. As a result, one could design $\psi$ using universal formulae as in (19). However, since $U$ is only a partial Lyapunov function, care should be taken to make sure that $r$ is bounded, and possibly converges to some value $r_{*}$. This motivates the following computations to get an alternative expression. Consider the inequality

$$
\begin{aligned}
& L_{g} V K L_{g} V^{T}+N L N^{T} \\
& =L_{g} V K L_{g} V^{T}+\left[L_{g} V-\frac{2 z}{r} L_{g} M\right] L\left[L_{g} V-\frac{2 z}{r} L_{g} M\right]^{T} \\
& \geq \frac{4 z^{2}}{r^{2}} L_{g} M L(K+L)^{-1} K L_{g} M^{T},
\end{aligned}
$$

and let

$$
\begin{aligned}
& P\left(x, \frac{z}{r}\right)= \\
& \quad L_{g} M(x) L\left(x, \frac{z}{r}\right)\left(K(x)+L\left(x, \frac{z}{r}\right)\right)^{-1} K(x) L_{g} M(x)^{T} .
\end{aligned}
$$

Since $K$ and $L$ are assumed to be positive definite, the matrix $L(K+L)^{-1} K$ is symmetric and positive definite as well. This yields

$$
\begin{aligned}
\overparen{U(x, y, r)} \leq & -N\left(x, \frac{z}{r}\right) L\left(x, \frac{z}{r}\right) N\left(x, \frac{z}{r}\right)^{T} \\
& -W(x)+\frac{2 z \delta(x)}{r}-\frac{z^{2}}{r^{2}}\left[\psi+4 P\left(x, \frac{z}{r}\right)\right] .
\end{aligned}
$$

We select $\psi$ as $\psi(x, z, r)=$

$$
\left\{\begin{array}{rr}
-4 P\left(x, \frac{z}{r}\right) \frac{r-r_{*}}{r} & \\
+\frac{\max \left\{-\alpha W(x)+\frac{2 z \delta(x)}{r}+\frac{z^{4}}{r^{4}} W(x), 0\right\} r^{2}}{z^{2}} & \text { if } z \neq 0 \\
-4 P(x, 0) \frac{r-r_{*}}{r} & \text { if } z=0,
\end{array}\right.
$$

where $\alpha$ is chosen in $(0,1)$ and $r_{*}$ is the nominal value we choose for $r$. It follows from (21) that this function is continuous on $\mathbb{R}^{n} \times \mathbb{R} \times \mathbb{R}_{+*}$. Note also that the expression of $\psi$ involves only known data. Observe that, for any $\beta$ in $(0,1]$, $\dot{r}$ is non-negative when $r \leq \beta r_{*}$, hence the set $r \geq \beta r_{*}$ is forward invariant for the closed-loop system.

To study the behavior of the solutions of the closed-loop system, we define the function

$$
\mathcal{U}(x, y, r)=\left[\log (r)+\frac{r_{*}}{r}\right]+\kappa(U(x, y, r)),
$$

where $\kappa$ is to be chosen as a $C^{1}$ class $\mathcal{K}^{\infty}$ function. $\mathcal{U}$ is positive definite in $\left(x, y, r-r_{*}\right)$ and proper on $\mathbb{R}^{n} \times \mathbb{R} \times \mathbb{R}_{+*}$. Its derivative along the solutions of the closed-loop system satisfies :

$$
\begin{aligned}
& \overparen{\mathcal{U}(x, y, r)} \leq \\
& +\frac{r-r_{*}}{r^{2}} \psi(x, z, r)-\kappa^{\prime}(U(x, y, r)) \times \\
& \quad \times\left[N\left(x, \frac{z}{r}\right) L\left(x, \frac{z}{r}\right) N\left(x, \frac{z}{r}\right)^{T}\right. \\
& \left.\quad+\left(\left[(1-\alpha)+\frac{z^{4}}{r^{4}}\right] W(x)+4 P\left(x, \frac{z}{r}\right) \frac{z^{2}}{r^{2}} \frac{r_{*}}{r}\right)\right] .
\end{aligned}
$$

Since, in the expression of $\psi$, we have, for $z \neq 0$,

$$
\frac{\max \left\{-\alpha W(x)+\frac{2 z \delta(x)}{r}+\frac{z^{4}}{r^{4}} W(x), 0\right\} r^{2}}{z^{2}} \leq\left[\frac{\ell^{\prime}(V(x))^{2}}{\alpha}+\frac{z^{2}}{r^{2}}\right] W(x),
$$

by completing squares, we obtain, for $r \geq \beta r_{*}$,

$$
\begin{aligned}
\frac{r-r_{*}}{r^{2}} \psi(x, z, r) & \leq-4 P\left(x, \frac{z}{r}\right) \frac{\left(r-r_{*}\right)^{2}}{r^{2}} \\
+ & {\left[\frac{\ell^{\prime}(V(x))^{2}}{\alpha \beta r_{*}}+\frac{z^{4}}{2 r^{4}}+\frac{1}{2 \beta^{2} r_{*}^{2}}\right] W(x) . }
\end{aligned}
$$

This yields, for $r \geq \beta r_{*}$,

$$
\begin{aligned}
& \overparen{\mathcal{U}(x, y, r)} \leq \\
& \quad-4 P\left(x, \frac{z}{r}\right)\left[\frac{\left(r-r_{*}\right)^{2}}{r^{2}}+\kappa^{\prime}(U(x, y, r)) \frac{z^{2}}{r^{2}} \frac{r_{*}}{r}\right] \\
& \quad-\kappa^{\prime}(U(x, y, r)) N\left(x, \frac{z}{r}\right) L\left(x, \frac{z}{r}\right) N\left(x, \frac{z}{r}\right)^{T} \\
& \quad-\left[(1-\alpha) \kappa^{\prime}(U(x, y, r))-\frac{\ell^{\prime}(V(x))^{2}}{\alpha \beta r_{*}}-\frac{1}{2 \beta^{2} r_{*}^{2}}\right] W(x) \\
& -\left[\kappa^{\prime}(U(x, y, r))-\frac{1}{2}\right] \frac{z^{4}}{r^{4}} W(x) .
\end{aligned}
$$

By condition (8), equations (17) and (22), and since $K$ 
and $L$ are positive definite, $\overparen{\mathcal{U}(x, y, r)}$ is negative definite in $\left(x, y, r-r_{*}\right)$ on $\mathbb{R}^{n} \times \mathbb{R} \times \mathbb{R}_{+*}$ provided $\kappa^{\prime}$ is chosen non decreasing and satisfying :

$$
\kappa^{\prime}(v) \geq \max \left\{\frac{1}{2}, \frac{2}{1-\alpha}\left[\frac{\ell^{\prime}(V(x))^{2}}{\alpha \beta r_{*}}+\frac{1}{2 \beta^{2} r_{*}^{2}}\right]\right\} .
$$

The inequality (23) is established only for $r \geq \beta r_{*}$ and $\kappa$ and therefore $\mathcal{U}$ depend on $\beta$. But $\beta$ is arbitrary in $(0,1]$ and the closed-loop system does not depend on this real number. Hence we have proved the asymptotic stability of the point $\left(0,0, r_{*}\right)$ with $\mathbb{R}^{n} \times \mathbb{R} \times \mathbb{R}_{+*}$ as domain of attraction.

Finally note that, also in this second design, in which no information on $\ell$ has been exploited, the control can be approximated, locally around the origin, as in equation (20).

C. Design III - $\ell$ unknown, $K$ non-positive definite, $\ell^{\prime}(0)$ known

In section III-A, the knowledge of $\ell$ allowed us to dominate the term $\frac{z \delta(x)}{r}$ with an appropriate choice of $\psi$. In section III-B, we took advantage of the negative definite term in $\frac{z}{r}$ provided by the definite negativeness of $K$. We consider now the case in which the function $\ell$ in Lemma 1 is unknown (although it exists) and $K$ may be singular. Fortunately, when $\ell^{\prime}(0)$ is known, it is possible to modify the technique introduced in Section III-A to obtain a global asymptotic stability result. To show this we assume that we know a pair of strictly positive real numbers $\left(v_{0}, \kappa_{0}\right)$ satisfying :

$$
\frac{2|\delta(x)|}{\left|L_{f} V(x)\right|}<\sqrt{\kappa_{0}} \quad \forall x: V(x) \in\left(0, v_{0}\right] .
$$

Then, let $\sigma: \mathbb{R}_{+} \rightarrow[0,1]$ be a $C^{1}$ function, with a nonpositive derivative, such that ${ }^{6}$

$$
\begin{aligned}
\sigma(v)=1 & \text { if } \quad v=0, \\
\in[0,1] & \text { if } \quad 0<v<1, \\
=0 & \text { if } \quad 1 \leq v,
\end{aligned}
$$

and define

$$
\begin{aligned}
\mathcal{U}_{l o c}(x, y, r)= & \lambda \sigma\left(\frac{U(x, y, r)}{v_{0}}\right) \frac{\left(r-r_{*}\right)^{2}}{1+\left(r-r_{*}\right)^{2}} \\
& +U(x, y, r),
\end{aligned}
$$

with $^{7}$

$$
\lambda<\min \left\{\frac{8 \alpha}{3 \sqrt{3} \kappa_{0}}, \frac{v_{0}}{2 \max _{v \in[0,1]}\left|\sigma^{\prime}(v)\right|}\right\},
$$

where $\alpha$ in chosen in $\left(0, \frac{1}{4}\right)$. As opposed to the functions $\mathcal{U}$ we introduced in sections III-A and III-B, $\mathcal{U}_{\text {loc }}$ is positive definite and proper only in $\left(x, \frac{z}{\sqrt{r}}\right)$. Therefore complementary arguments are needed to derive a stability result. Nevertheless with the nominal control $u$ given in (18),

\footnotetext{
${ }^{6}$ For example, we could select $\sigma(v)=2(1-v)^{2}-(1-v)^{4}$ for $v \in[0,1]$ and $=0$ elsewhere.

${ }^{7}$ The bound is obtained observing that the derivative of the function $\frac{r^{2}}{1+r^{2}}$ is upper-bounded by $\frac{3 \sqrt{3}}{8}$.
}

this functions gives

$$
\begin{gathered}
\overbrace{\mathcal{U}_{l o c}(x, y, r)}= \\
\quad\left(\lambda \sigma\left(\frac{U(x, y, r)}{v_{0}}\right) \frac{2\left(r-r_{*}\right)}{\left(1+\left(r-r_{*}\right)^{2}\right)^{2}}-\frac{z^{2}}{r^{2}}\right) \psi \\
\quad-\left[\frac{\lambda}{v_{0}} \sigma^{\prime}\left(\frac{U(x, y, r)}{v_{0}}\right) \frac{\left(r-r_{*}\right)^{2}}{1+\left(r-r_{*}\right)^{2}}+1\right] \times \\
\times\left[\left|L_{f} V(x)\right|+2 N\left(x, \frac{z}{r}\right) L\left(x, \frac{z}{r}\right) N\left(x, \frac{z}{r}\right)^{T}-\frac{2 z \delta(x)}{r}\right] .
\end{gathered}
$$

By completing squares and using the properties

$$
\begin{gathered}
\frac{2\left(r-r_{*}\right)}{\left(1+\left(r-r_{*}\right)^{2}\right)^{2}} \leq \frac{3 \sqrt{3}}{8} \leq \frac{\alpha}{\lambda \kappa_{0}}, \\
\sigma\left(\frac{U(x, y, r)}{v_{0}}\right)=0 \quad \Rightarrow \quad|\delta(x)|^{2} \leq \kappa_{0}\left|L_{f} V(x)\right|^{2},
\end{gathered}
$$

we have

$$
\begin{aligned}
-2 \sqrt{\alpha} \mid L_{f} V & (x) \mid+\frac{2 z \delta(x)}{r} \\
& \leq-\sqrt{\alpha}\left|L_{f} V(x)\right|+\frac{\delta(x)^{2}}{\sqrt{\alpha}\left|L_{f} V(x)\right|} \frac{z^{2}}{r^{2}} \\
& \leq \frac{\delta(x)^{2}}{\sqrt{\alpha}\left|L_{f} V(x)\right|} \times \\
& \times\left(\frac{z^{2}}{r^{2}}-\lambda \sigma\left(\frac{U(x, y, r)}{v_{0}}\right) \frac{2\left(r-r_{*}\right)}{\left(1+\left(r-r_{*}\right)^{2}\right)^{2}}\right)
\end{aligned}
$$

In addition the selection of $\lambda$ is such that

$$
\frac{3}{2} \geq\left[\frac{\lambda}{v_{0}} \sigma^{\prime}\left(\frac{U(x, y, r)}{v_{0}}\right) \frac{\left(r-r_{*}\right)^{2}}{1+\left(r-r_{*}\right)^{2}}+1\right] \geq \frac{1}{2} .
$$

By (12), this proves that, with $\psi$ as control, $\mathcal{U}_{\text {loc }}$ satisfies the property of being a control Lyapunov function ${ }^{8}$ and the small control property. Hence $\psi$ can be selected using the universal formula (19) with

$$
\begin{aligned}
A=\left[2 \sqrt{\alpha} \mid L_{f}\right. & \left.V(x) \mid-\frac{2 z \delta(x)}{r}\right] \times \\
& \times\left[\frac{\lambda}{v_{0}} \sigma^{\prime}\left(\frac{U(x, y, r)}{v_{0}}\right) \frac{\left(r-r_{*}\right)^{2}}{1+\left(r-r_{*}\right)^{2}}+1\right]
\end{aligned}
$$

and

$$
B=\lambda \sigma\left(\frac{U(x, y, r)}{v_{0}}\right) \frac{2\left(r-r_{*}\right)}{\left(1+\left(r-r_{*}\right)^{2}\right)^{2}}-\frac{z^{2}}{r^{2}}
$$

As a result

$$
\begin{aligned}
\overparen{\mathcal{U}_{l o c}(x, y, r)} & \leq-\mathfrak{W}_{l o c}\left(x, \frac{z}{r}\right) \\
& -\left(\lambda \sigma\left(\frac{U(x, y, r)}{v_{0}}\right) \frac{2\left(r-r_{*}\right)}{\left(1+\left(r-r_{*}\right)^{2}\right)^{2}}-\frac{z^{2}}{r^{2}}\right)^{2}
\end{aligned}
$$

where

$$
\mathfrak{W}_{l o c}=\frac{1}{2}\left((1-2 \sqrt{\alpha})\left|L_{f} V(x)\right|+2 N\left(x, \frac{z}{r}\right) L\left(x, \frac{z}{r}\right)\right)
$$

${ }^{8}$ Although as already mentioned it is not positive definite and proper in $(x, y, r)$. 
is a positive definite in $\left(x, \frac{z}{r}\right)$. We take advantage of this positiveness and modify the function $\psi$ defined above by adding the term

$$
-\beta \mathfrak{W}_{l o c}\left(x, \frac{z}{r}\right) \frac{r}{1+z^{2}}\left(r-r_{*}\right),
$$

with $\beta$ in $(0,1)$. This term remains strictly positive as $r$ goes to 0 except if $x$ and $\frac{z}{r}$ goes to 0 too. But the remaining part of $\psi$ given by the universal formula (19) tends to $\frac{2 \lambda r_{*}}{\left(1+r_{*}^{2}\right)^{2}}$ as $\left(x, \frac{z}{r}, r\right)$ tends to 0 . So the set $r>0$ is forward invariant for the closed-loop system. Then, since we have

$$
\begin{aligned}
-\beta \mathfrak{W}_{l o c} & \left(x, \frac{z}{r}\right) \frac{r}{1+z^{2}}\left(r-r_{*}\right) \times \\
& \times\left(\lambda \sigma\left(\frac{U(x, y, r)}{v_{0}}\right) \frac{2\left(r-r_{*}\right)}{\left(1+\left(r-r_{*}\right)^{2}\right)^{2}}-\frac{z^{2}}{r^{2}}\right) \leq \beta \mathfrak{W}_{l o c},
\end{aligned}
$$

this modification yields

$$
\begin{aligned}
\overbrace{\mathcal{U}_{l o c}(x, y, r)} & \leq-(1-\beta) \mathfrak{W}_{l o c}\left(x, \frac{z}{r}\right) \\
& -\left(\lambda \sigma\left(\frac{U(x, y, r)}{v_{0}}\right) \frac{2\left(r-r_{*}\right)}{\left(1+\left(r-r_{*}\right)^{2}\right)^{2}}-\frac{z^{2}}{r^{2}}\right)^{2} .
\end{aligned}
$$

It follows that, along any closed-loop system solution, as long as it exists in positive time, $x, \frac{z}{r}, \phi\left(x, \frac{z}{r}\right)$ in (18) and, therefore $\dot{x}$ and $\dot{z}$, are bounded and

$$
\left(\lambda \sigma\left(\frac{U(x, y, r)}{v_{0}}\right) \frac{2\left(r-r_{*}\right)}{\left(1+\left(r-r_{*}\right)^{2}\right)^{2}}-\frac{z^{2}}{r^{2}}\right)^{2}
$$

and $\mathfrak{W}_{l o c}\left(x, \frac{z}{r}\right)$ are integrable. As a result, $\dot{r}$ is upperbounded along any solution and all solutions are defined in $[0,+\infty)$. It follows that $\lambda \sigma\left(\frac{U(x, y, r)}{v_{0}}\right) \frac{2\left(r-r_{*}\right)}{\left(1+\left(r-r_{*}\right)^{2}\right)^{2}}-\frac{z^{2}}{r^{2}}, x$ and $\frac{z}{r}$ tend to 0 , as time goes to $\infty$. Finally, since $\sigma(0)=1$, we have also that $r$ converges to $r_{*}$.

\section{Conclusions}

The stabilization problem for a simple class of feedforward systems has been revisited. It has been shown that the use of dynamically scaled control Lyapunov functions yields new, dynamic, state feedback, stabilizing control laws. To prove our results we have given expressions for the feedback which can be evaluated with the knowldege that we have on the system. In applications specific expressions should be derived.

\section{REFERENCES}

[1] D. Carnevale and A. Astolfi. Integrator forwarding without PDEs. In 48th Conference on Decision and Control, Shanghai, China, pages 33-38, 2009.

[2] R.A. Freeman and P.V. Kokotovic. Inverse optimality in robust stabilization. SIAM J. Control and Optimization, pages 1365-1391, 1996.

[3] M. Jankovic, R. Sepulchre, and P.V. Kokotovic. Constructive Lyapunov stabilization of nonlinear cascade systems. IEEE Trans. Autom. Control, 41:1723-1735, December 1996.

[4] D. Karagianis, M. Sassano, and A. Astolfi. Dynamic scaling and observer design with application to adaptive control. Automatica, 45:2883-2889, 2009.

[5] P. Krishamurthy and F. Khorrami. Generalized state scaling-based robust control of nonlinear systems and applications to tirangular systems. In American control Conference, Portland, OR, pages 34273432, 2005.
[6] F. Mazenc and L. Praly. Adding integrations, saturated controls, and stabilization for feedforward systems. IEEE Trans. Autom. Control, 41:1559-1578, November 1996.

[7] L. Praly, D. Carnevale, and A. Astolfi. Dynamic vs static scaling: an existence result. In IFAC Symposium on Nonlinear Control System Design, Bologna, IT, 2010.

[8] E.D. Sontag. A "universal" construction of Artstein's theorem on nonlinear stabilization. Systems and Control Letters, 13:117-123, 1989.

[9] A. R. Teel. Semi-global stabilization of minimum phase nonlinear systems in special normal forms. Systems and Control Letters, 19:187192, 1992.

[10] A. R. Teel. A nonlinear small gain theorem for the analysis of control systems with saturation. IEEE Trans. Autom. Control, 41:1256-1270, September 1996.

[11] Y. Xudong. Universal stabilization of feedforward nonlinear systems. Automatica, 39:141-147, 2004.

\section{APPENDIX I}

\section{PROOF OF LEMMA 1}

Let $\mathcal{L}: \mathbb{R}_{+*} \rightarrow \mathbb{R}$ be the function defined by :

$$
\mathcal{L}(v)=\sup _{x}: V(x) \leq v \frac{\delta(x)}{\left|L_{f} V(x)\right|} \quad v>0 .
$$

This function is well defined. If not, we would have a strictly positive real number $v_{*}$ and a sequence $x_{k}$ in $\mathbb{R}^{n}$ satisfying :

$$
V\left(x_{k}\right) \leq v_{*} \quad, \quad \frac{\delta\left(x_{k}\right)}{\left|L_{f} V\left(x_{k}\right)\right|} \geq n .
$$

Since $V$ is continuous and radially unbounded, $x_{k}$ is in a compact set. Let $x_{*}$ be any of its cluster points. By condition (7), $x_{*}$ cannot be the origin since it would contradict the unboundedness of the sequence $\frac{\delta\left(x_{k}\right)}{\left|L_{f} V\left(x_{k}\right)\right|}$. Then, $\left|L_{f} V\right|$ being positive definite, $\left|L_{f} V\left(x_{*}\right)\right|$ is non-zero and the function $\delta$ being continuous, the sequence $\frac{\delta\left(x_{k}\right)}{\left|L_{f} V\left(x_{k}\right)\right|}$ must be bounded, which is a contradiction.

From its definition, the function $\mathcal{L}$ satisfies :

$$
\mathcal{L}(V(x)) \geq \frac{\delta(x)}{\left|L_{f} V(x)\right|} \quad \forall x \in \mathbb{R}^{n} \backslash\{0\} .
$$

In addition, it is non-decreasing, hence it has a limit when $v$ tends to 0 . Let

$$
\mathcal{L}(0)=\lim _{v \searrow 0} \mathcal{L}(v) .
$$

Note now that, since $\delta$ and $L_{f} V$ are both zero at the origin, we have :

$$
\mathcal{L}(0)\left|L_{f} V(0)\right| \geq \delta(0) .
$$

Finally, the definition of $\mathcal{L}(0)$ implies that $\mathcal{L}$ is continuous at 0 . Actually $\mathcal{L}$ is continuous on $\mathbb{R}_{+}$. But proving this is long and we prefer the shortest path of defining the derivative $\ell^{\prime}$ of $\ell$ as a smoothed version of $\mathcal{L}$. Specifically, we define $\ell^{\prime}$ as the Riemann integral :

$$
\begin{aligned}
\ell^{\prime}(v) & =\frac{1}{v} \int_{v}^{2 v} \max \{w, \mathcal{L}(w)\} d w & & \text { if } v \neq 0, \\
& =\mathcal{L}(0) & & \text { if } v=0 .
\end{aligned}
$$

It is continuous, non-decreasing, non-integrable and satisfies :

$$
\mathcal{L}(v) \leq \ell^{\prime}(v)
$$

and therefore (12) holds. 